\title{
Nanolithography on the Electron Beam Resist using the Scanning Probe Microscope Cantilever
}

\author{
Lydia Anggraini $^{1}$, Naoki Matsuzuka ${ }^{2}$ \& Yoshitada Isono ${ }^{3}$ \\ ${ }^{1}$ Graduate School of Science and Engineering \\ ${ }^{2}$ College of Science and Engineering, Ritsumeikan University, \\ 1-1-1 Nojihigashi, Kusatsu, Shiga 525-8577, Japan \\ ${ }^{3}$ Graduate School of Engineering, Kobe University, \\ 1-1 Rokkodai-cho, Nada-ku, Kobe, Hyogo 657-8501, Japan \\ Email: gr040069@ed.ritsumei.ac.jp
}

\begin{abstract}
This research demonstrates the feasibility of fabricating nanoscale resist patterns on a silicon $(\mathrm{Si})$ substrate using a scanning probe microscope (SPM). In order to utilize scanning probe nanolithography (SPNL) with the other micro-machining techniques such as dry etching, plating and lift-off process, nanoscale resist patterns should be created on an Si substrate with high accuracy in SPNL. We have, so far, established the negative type of SPNL using the negative-tone electron beam (EB) resist named SAL601. The primary objective of this research is to find out appropriate process conditions for establishing the positive type of SPNL using the positive EB resist "ZEP520A". This paper describes the variations of experimentally created nano-patterns depending on the process conditions, and determines the appropriate process conditions from the variations obtained. In addition, we analyzed the electric field in the EB resist by a finite element method (FEM), for estimating the line width of the nanopattern created by SPNL.
\end{abstract}

Keywords: Direct nano-patterning; Positive-tone electron beam (EB) resist; Scanning probe microscope (SPM); Scanning probe nanolithography (SPNL).

\section{$1 \quad$ Introduction}

Establishing fabrication technique on a nanoscale is essential to downscaling silicon-based micro electromechanical systems (MEMS) for improving their performance, integration density, and power consumption. Nanoscale patterns have been created on a silicon ( $\mathrm{Si}$ ) substrate by state-of-the-art nanolithographic techniques such as electron beam (EB) lithography, extreme ultraviolet (EUV) lithography and X-ray lithography. EB lithography is generally utilized for creating nano-patterns among these techniques, because the technique has advantages of high resolution and repeatability. However, EB lithography might be difficult to be promising for trial manufacturing of MEMS devices in $\mathrm{R} \& \mathrm{D}$, since the initial cost is extremely high for small-scale laboratories.

Received April $4^{\text {th }}, 2009$, Revised August $20^{\text {th }}, 2009$, Accepted for publication October $15^{\text {th }}, 2009$. 
Scanning probe nanolithography (SPNL) is a very promising technique for creating nano-patterns on an $\mathrm{Si}$ substrate with comparative low cost, because SPNL is based on a scanning probe microscope (SPM) [1-6]. The resolution of SPNL stands comparison with that of EB lithography. Furthermore, a throughput of SPNL will be improved by the development of a parallel writing technique with multi-probe cantilever array [7,8]. SPNL produces silicon dioxide $\left(\mathrm{SiO}_{2}\right)$ patterns with less than $100 \mathrm{~nm}$ in width, based on field-enhanced anodization. However, SPNL using a resist is much preferable to combine with the other micro-machining techniques such as dry etching, plating and lift-off process. Among various kinds of resists, EB resists are suitable for SPNL that injects electrons from a tip of an SPM probe into the resist, because molecule of EB resist chemically reacts with the electrons. We have, so far, established the negative type of SPNL using a negative-tone EB resist SAL601 [6].

The primary objective of this research is to establish positive type of SPNL using positive-tone EB resist for creating a nanoscale groove on the resist formed on an Si substrate. This research used "ZEP520A" commercialized by ZEON CORPORATION as a positive EB resist for SPNL. The EB resist was chosen because this resist has the high performance for resolution, sensitivity and dry etch resistance [9]. We experimentally investigated appropriate process conditions of the pre-bake and electron exposure procedures. In addition, we analyzed the electric field in the resist by a finite element method, for estimating the line width of the nano-pattern created by SPNL.

\section{Experimental Method}

Figure 1 shows a procedure of positive type of SPNL with ZEP520A. The process starts from a formation of a thin ZEP520A layer on an Si substrate. After the formation of the resist, a positive bias voltage is applied to the $\mathrm{Si}$ substrate and a conductive probe is connected to ground (GND), which emits electrons from the tip of the probe to the resist layer. Nano-patterning is carried out by scanning the probe on the resist surface with applying the bias voltage. Finally, the nanoscale groove is created on the resist surface after a development process.

The fabrication process starts with bulk silicon ( $\mathrm{Si}$ ) wafers with a thickness of $525 \mu \mathrm{m}$. Before depositing the EB-resist on the Si substrate using the spin coat, the dehydration bake process is needed. The preparation process start from the dehydration bake on the hot plate with bake temperature of $110{ }^{\circ} \mathrm{C}$ for 10 minutes, the purpose of this process is to remove water molecule on the substrate. We diluted ZEP520A with an organic solvent in order to form a thin 


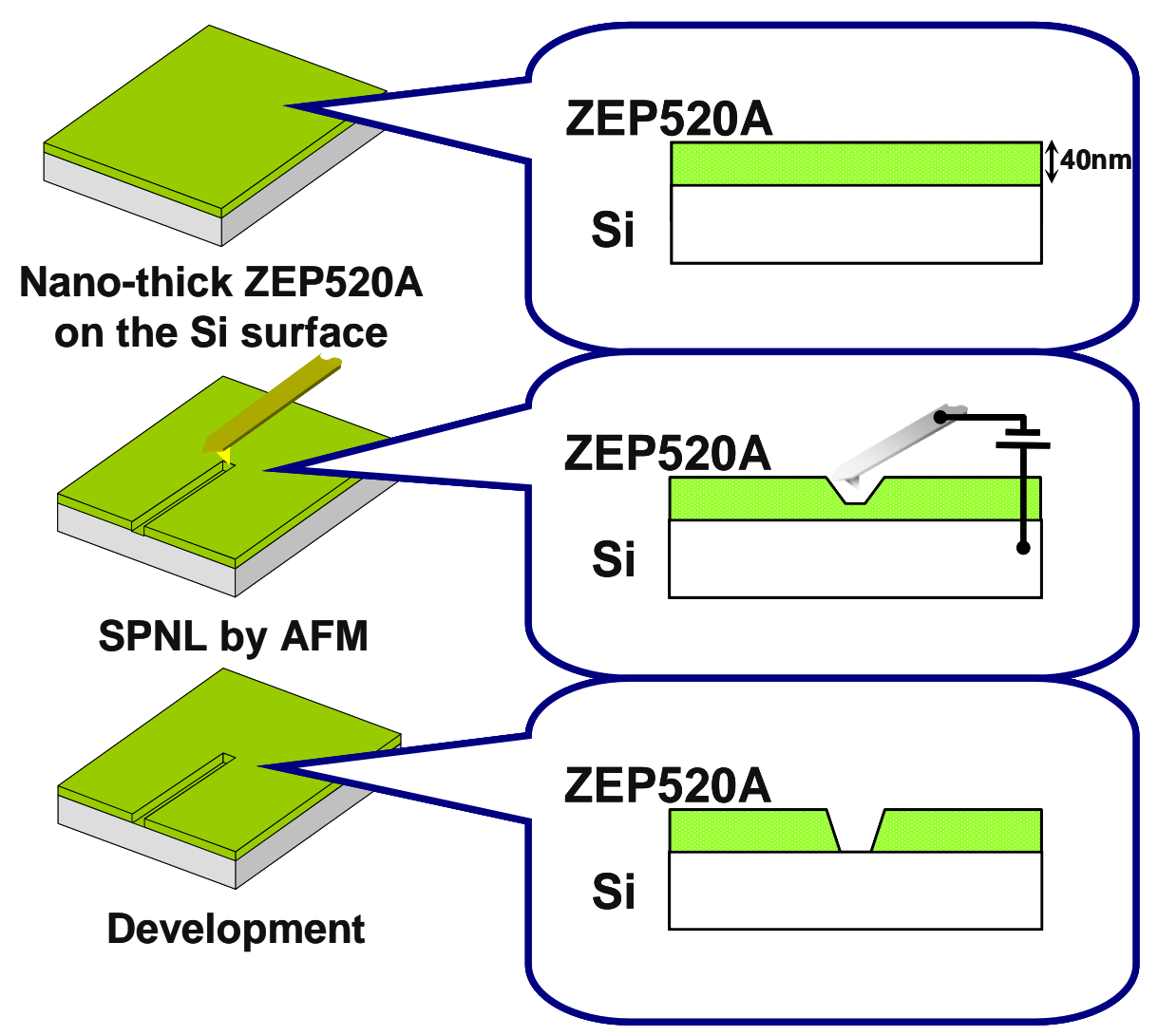

Figure 1 Fabrication processes of SPNL using positive-tone EB resist

resist layer on a substrate and realize very fine patterns. The concentration of the resist used is $33 \mathrm{wt} \%$, which realizes a $40 \mathrm{~nm}$-thick resist layer under the spin-coating condition of $1 \mathrm{st}-300 \mathrm{rpm}$ for $3 \mathrm{sec}$ and 2 nd-5000 rpm for $120 \mathrm{sec}$. After depositing the EB-resist by the spin coat, the next process is pre-bake. The purpose of pre-bake process is that the adhesion between the Si substrate and the EB-resist is improved after coating process. This process is one of the most important points of SPNL technology, because a good surface roughness can be obtained on the profile. The pre-bake of the resist is carried out on a hot plate at $180{ }^{\circ} \mathrm{C}$ temperature. After the bake, the subsequent electron exposure is carried out using an SPM. The electron exposure is a very important process for the quality of the nano-patterns created. Here, we can optimize the process parameters such as the applied bias voltage, scan speed and scan time. We used the SPM and probe cantilever coated by a gold (Au) layer commercialized by Seiko Instruments Inc, (SII) which has a tip radius around $20 \mathrm{~nm}$. The bias voltage was applied to the sample stage from 32 to $40 \mathrm{~V}$ by $2 \mathrm{~V}$ step. The scan 
speeds of the cantilever are determined to $0.1,1.0$ and $2.0 \mu \mathrm{m} / \mathrm{sec}$ for this study. The scan time was determined to 10 times, because the sensitivity of positivetone EB resists is generally lower than that of negative-tone EB resist. After the electron exposure, the nano-thickness of EB-resists is dipped with a substrate into a developer ZED-N50, and then is rinsed by ZMD-B. Finally, post-bake is carried out on a hot plate at $110{ }^{\circ} \mathrm{C}$ temperature. The nano-groove created is evaluated by an SPM.

Furthermore, the characterization technique through the EB resist was measured during the writing procedure using the current monitoring system installed in the SPM. The applied voltage was increased from $20 \mathrm{~V}$ by the step of $1 \mathrm{~V}$. The scan speed of 1.0 and $2.0 \mu \mathrm{m} / \mathrm{sec}$ were also tested to confirm the difference of the currents depending on the scan speed.

\section{$3 \quad$ Results and Discussions}

\subsection{Experimental Results of Scanning Probe Nanolithography using Positive EB resist}

Nano-metric ZEP520A resist were coated on Si surface using the spin coating. The pre-bake time was carried out at $2 \mathrm{~min}, 3 \mathrm{~min}$ and $5 \mathrm{~min}$ using a hot-plate at the temperature of $180^{\circ} \mathrm{C}$. The single probe of SPM cantilevers is used to expose the EB-resist surface. Figure 2 shows SPM images of nano-grooves created under the applied bias voltage from 32 to $40 \mathrm{~V}$, scan speed of $0.1,1.0$ and $2.0 \mu \mathrm{m} / \mathrm{sec}$. When the scan speed was $0.1 \mu \mathrm{m} / \mathrm{sec}$, deformation of the resist was observed at the voltage of 36,38 and $40 \mathrm{~V}$. The tip of the probe was damaged at these voltages, this caused by a large number of electrons were emitted from the tip, and at the scan speed of $0.1 \mu \mathrm{m} / \mathrm{sec}$ the moving tip is very slow. Therefore, the electrons gathered in one area, these phenomena make the tip of the probe can be easy damaged. Compared with the scan speeds of 1.0 and $2.0 \mu \mathrm{m} / \mathrm{sec}$, the opening widths of the nano-grooves were almost equal or less under the same bias voltage. The depths of the nano-grooves obtained by $2.0 \mu \mathrm{m} / \mathrm{sec}$ were different from the results of $1.0 \mu \mathrm{m} / \mathrm{sec}$ scan speed, and are not enough. Therefore, the scan speed of $1.0 \mu \mathrm{m} / \mathrm{sec}$ was preferable compared with $2.0 \mu \mathrm{m} / \mathrm{sec}$. We succeeded in creating the nano-grooves with the width from 50 to $130 \mathrm{~nm}$ at the scan speed of $1.0 \mu \mathrm{m} / \mathrm{sec}$. However, the depth decreased with a decrease of the width. In order to utilize the positive type of SPNL in a MEMS fabrication, the depth should reach to a surface of an Si substrate. The initial thickness of the resist was around $40 \mathrm{~nm}$ with the diluted resist of $33 \mathrm{wt} \%$. The initial thickness of should be decreased if the width needs to be less than 100 nm. 


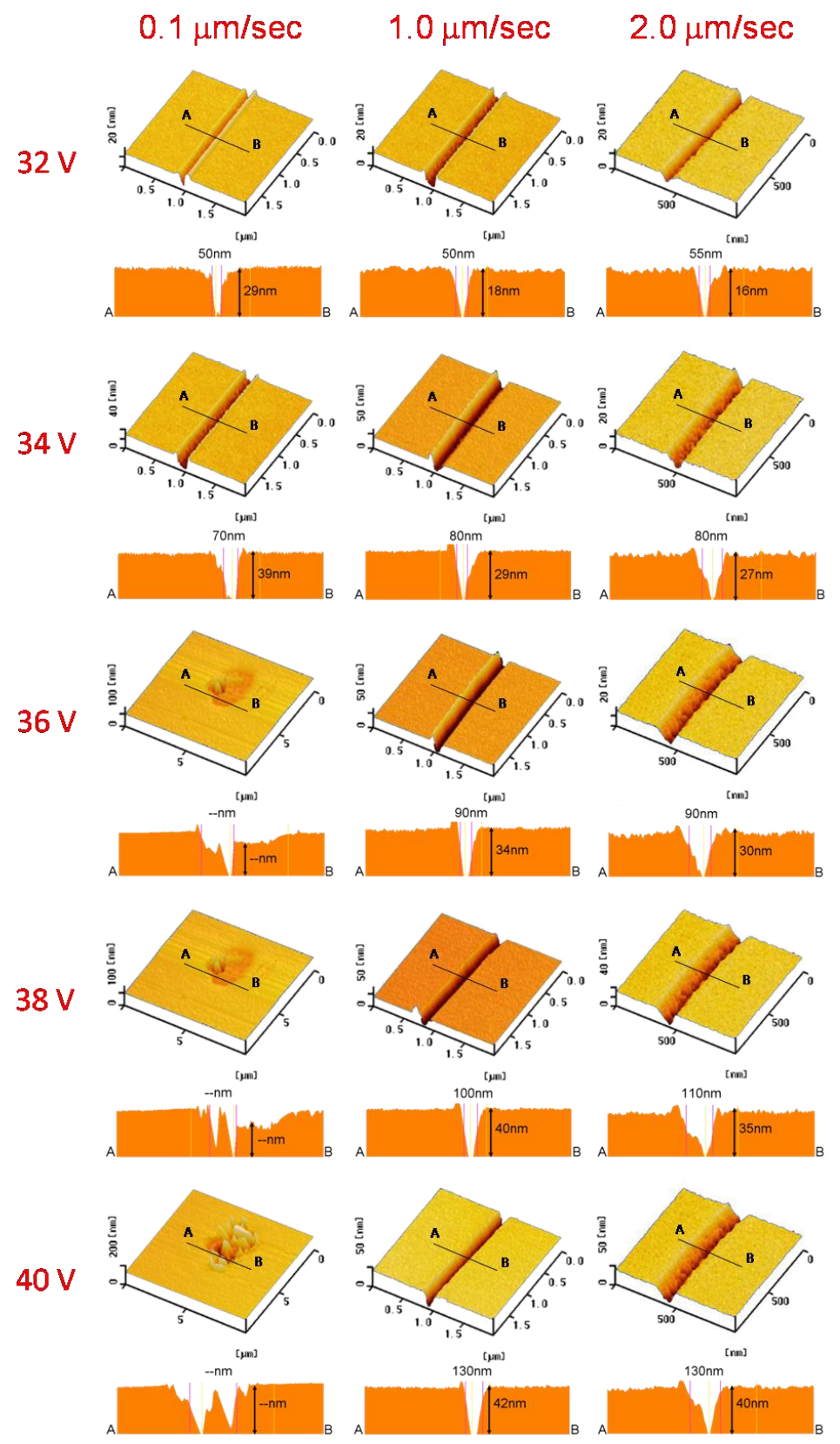

Figure 2 SPM images of nano-grooves patterned by positive SPNL at a prebake time of $5 \mathrm{~min}$ and multiple (10) scan. 
The effect of applied voltages on the pattern size of ZEP520A nano groove depth fabricated by a single probe cantilevser of SPM at a constant scan speed of $0.1,1.0$ and $2.0 \mu \mathrm{m} / \mathrm{sec}$ obtained from positive SPNL is shown in Figure 3. The effect of applied voltages strongly influenced the groove depth, since the depth increased linearly, this caused by the gaining energy from the tip cantilever resulted that the depth increased with an increase of the applied voltages. The deeper grooves were obtained at a higher applied voltage. The average line groove of positive SPNL at 1.0 and $2.0 \mu \mathrm{m} / \mathrm{sec}$ scan speed obtained a nano groove depth from 18 to $42 \mathrm{~nm}$ and 16 to $40 \mathrm{~nm}$ at applied voltages from $32 \mathrm{~V}$ to $40 \mathrm{~V}$, respectively.

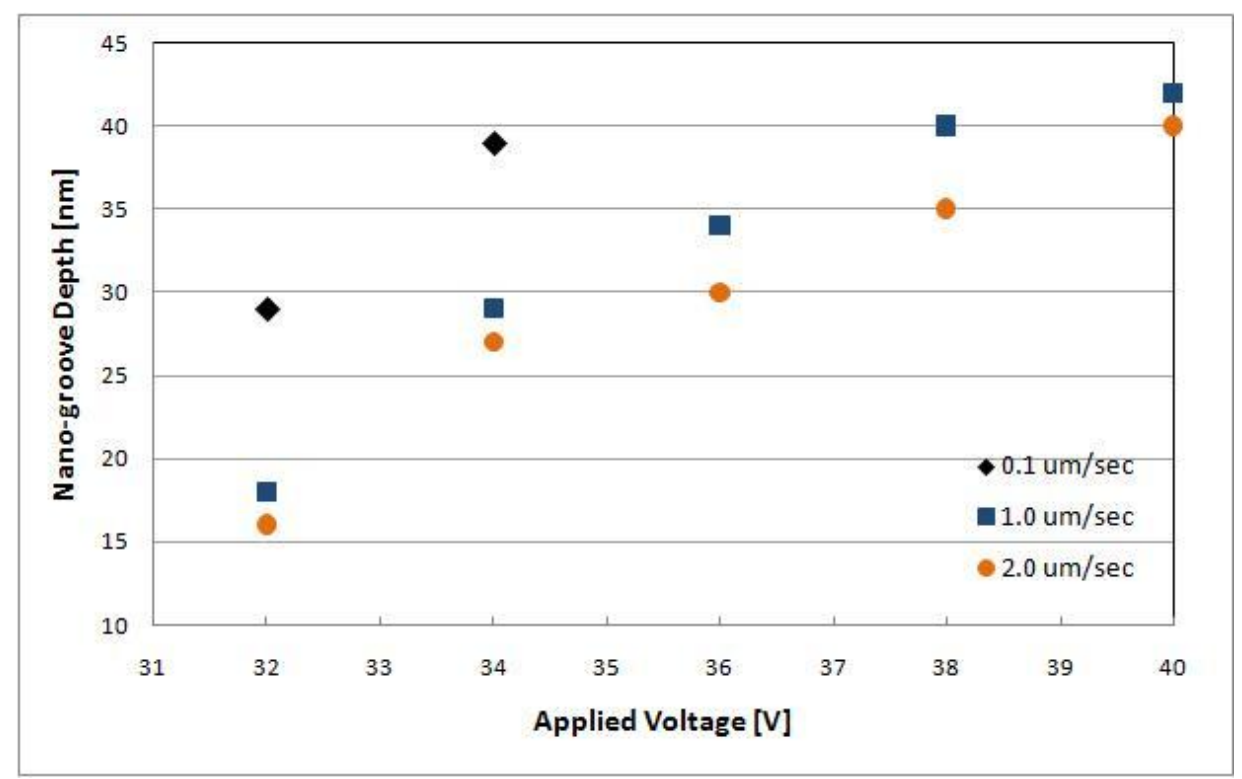

Figure 3 Effect of applied voltage on the depth of nano groove patterned by positive SPNL using EB-resist at a scan speed 0.1, 1.0, and $2.0 \mu \mathrm{m} / \mathrm{sec}$.

\subsection{Characteristics of Positive EB resist}

The quality of nano-patterns, i.e. width and depth, is determined by the current from the tip into the resist because of the characteristics of an EB resist. Hence, it is very important for us to obtain the information of the current.

Figure 4 shows the measured current-voltage (I-V) characteristics when scan speed was 1.0 and $2.0 \mu \mathrm{m} / \mathrm{sec}$. Each $\mathrm{I}-\mathrm{V}$ characteristic is divided into three regions: (I) $\mathrm{I}-\mathrm{V}$ characteristic follows the $\mathrm{Ohm}$ 's law, (II) current steeply increases with an increase of the bias voltage, and (III) large current is suddenly emitted. In the region (I), the current measured was $10 \mathrm{pA}$ or below. In the 
region (II), the currents measured were from 9 to $15 \mathrm{pA}$ and from 7 to $10 \mathrm{pA}$ at the scan speed of 1.0 and $2.0 \mu \mathrm{m} / \mathrm{sec}$, respectively. The enough depth of the nano-groove was obtained under the condition that the bias voltage was from 36 to $40 \mathrm{~V}$ and the scan speed was $1.0 \mu \mathrm{m} / \mathrm{sec}$. The currents under those conditions were from 10 to $15 \mathrm{pA}$. In the region (III), the large current was suddenly flown, and the tip of the probe was damaged. In detail, an Au layer around the tip came off the probe. This phenomenon was observed when we applied the bias voltages over $36 \mathrm{~V}$ at the scan speed of $0.1 \mu \mathrm{m} / \mathrm{sec}$. Even if the scan speed was $1.0 \mu \mathrm{m} / \mathrm{sec}$, this phenomenon was sometimes observed around $40 \mathrm{~V}$, depending on the probe cantilever. It is considered that a large number of electrons were emitted from the probe because of the local electric field concentration around the sharp tip, and then electro migration was occurred.

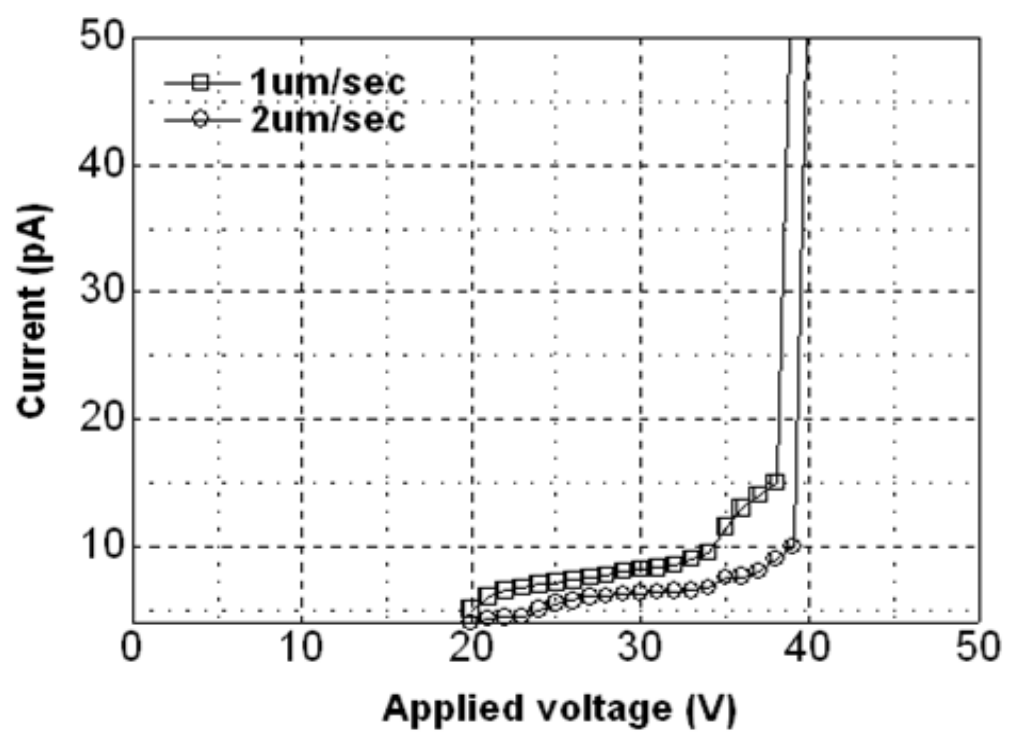

Figure 4 I-V characteristics of the positive SPNL using ZEP520A resist.

From the experimental results of positive SPNL, the successful range of the bias voltage for nano-patterning was from 36 to $40 \mathrm{~V}$ at $1.0 \mu \mathrm{m} / \mathrm{sec}$. Then, the current was estimated around $10 \mathrm{pA}$ from Figure 4. The electromigration was occurred around $40 \mathrm{~V}$, which is closed to the required bias voltage to successful nano-patterning. Therefore, the characterization results shows that the scan speed of $1.0 \mathrm{~mm} / \mathrm{sec}$ is appropriate to use to developed nano pattern. Furthermore, the careful current control is required to avoid the risk of the electromigration in positive SPNL. 


\subsection{Analytical Results}

Nanopattern of EB resists includes the sharp tip of SPM cantilever has been assumed in the analytical model. Finite-element modeling used for all of the simulations presented here were automatically generated using MSC Marc Mentat preprocessor software package. The analytical model consists of three kinds of material, Au tip cantilever, EB resist, and silicon. As a purpose of the analytical results of electric field intensity is the estimation of the widths on the nano groove.

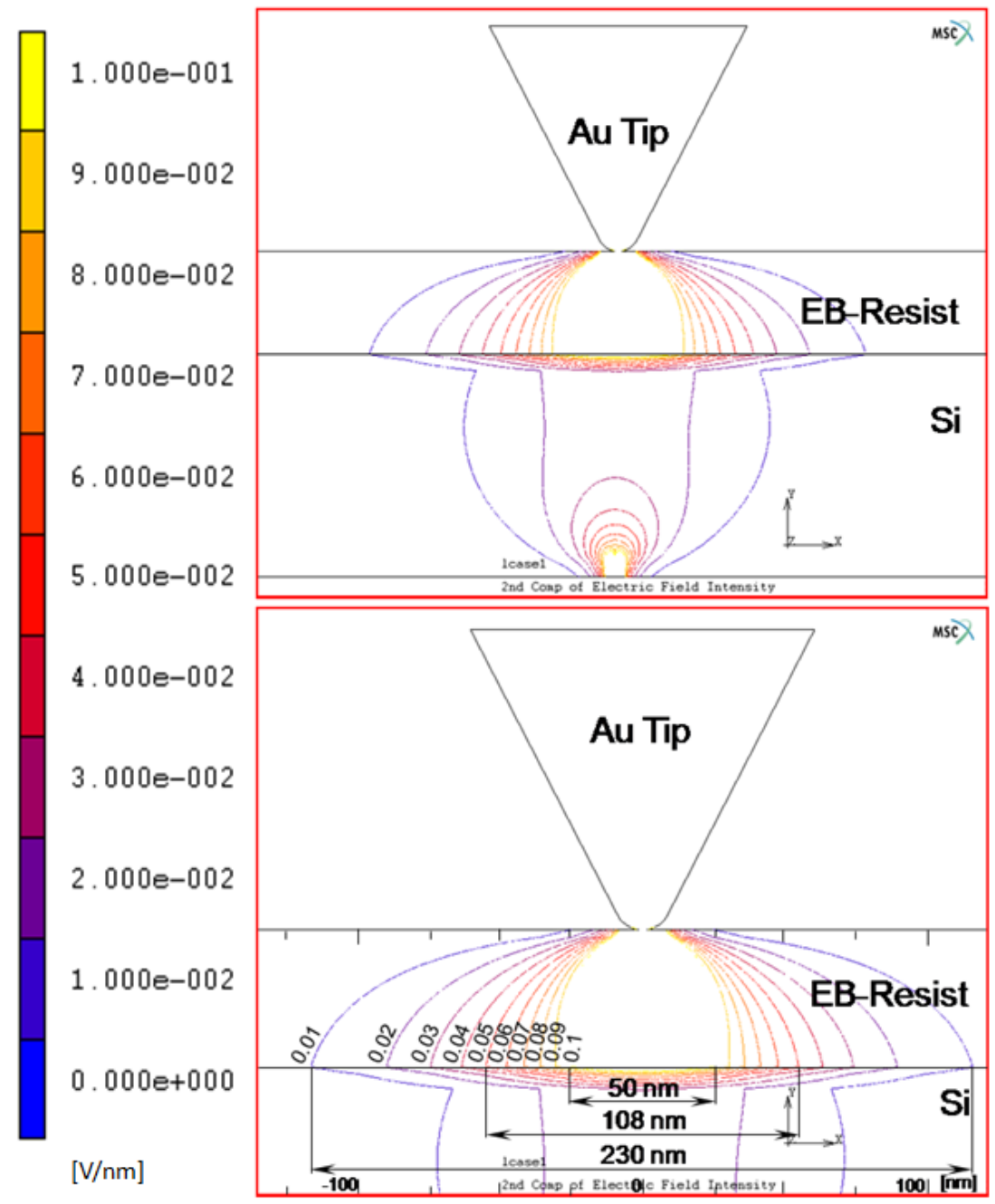

Figure 5 Analytical results with a contour line of electric field intensity inside EB resists at a total charge of $-15 \mathrm{pC}$. 
The electric field intensity was estimated by analytical results, in order to investigate the effect of electric distribution on the nanopattern size. As a model, a spherical tip with a radius of $10 \mathrm{~nm}$ was assumed. The radius of the contact region of $1.5 \mathrm{~nm}$ and the penetration depth of the probe tip into the resist, $0.227 \mathrm{~nm}$, were also assumed based on the Hertz equation.

Figure 5 shows the calculation results of analytical model and the contour line of electric field intensity at a total charge $-15 \mathrm{pC}$. Furthermore, the maximum width of electric field intensity of $0.05 \mathrm{~V} / \mathrm{nm}$ at a total charge $-15 \mathrm{pC}$ is $108 \mathrm{~nm}$. The positive EB resist was obtained at $15 \mathrm{pA}$ from the $\mathrm{I}-\mathrm{V}$ characteristics result, which approximately equal to applied bias voltage at $38 \mathrm{~V}$ (Figure 4). So, we analyzed this sample to compare with the experimental results. On the experimental results of nano-lines width of positive SPNL by using a constant scan speed $1 \mu \mathrm{m} / \mathrm{sec}$ and applied bias voltage $38 \mathrm{~V}$ is $100 \mathrm{~nm}$ (Figure 2). Therefore, we can conclude that the experimental results of positive SPNL using EB resist is near to the analytical results of electric field intensity of 0.05 $\mathrm{V} / \mathrm{nm}$. The distribution of the energy gained by an electron is proportional to the distribution of the electric field intensity. The electrons were field-emitted from the tip and most of the energy from the applied voltage was given to the electrons before they reached the resist surface. The electron those are fieldemitted from the tip into the EB resist, lose mainly along the electric field intensity while gaining energy from the electric potential.

\section{Conclusions}

This research demonstrated the feasibility of fabricating nanoscale resist patterns on an Si substrate using an SPM. In this research, we experimentally investigated the appropriate process conditions for the pre-bake time, applied bias voltage and scan speed, for the positive type of SPNL with positive-tone EB resist ZEP520A.

From the experimental results, the most appropriate process condition was determined to the applied bias voltages from 36 to $40 \mathrm{~V}$ and scan speed of 1.0 $\mu \mathrm{m} / \mathrm{sec}$. This process condition realized the nano-groove that has the opening width of $100 \mathrm{~nm}$ and the depth of $40 \mathrm{~nm}$. In addition, the current emitted was measured under the process condition. As a result, it was found that the range of the appropriate current was from 10 to $15 \mathrm{pA}$ for obtaining the groove depth around $40 \mathrm{~nm}$, which realized the $100 \mathrm{~nm}$ in width.

Finally, the electric field intensity was calculated by analytical results, in order to estimate the widths on the nanopattern size of EB resists. From these results, 
the SPNL process using an EB resists was established, and it could be expected that the SPNL process can provide greater sizes for nano fabrication. Finally, the applications to nano devices by using this technology are also expected.

\section{Acknowledgements}

The authors would like to thank to the reviewers for useful comments and suggestions about carrying this journal.

\section{References}

[1] Dagata, J.A., Schneir, J., Harary, H.H., Evans, C.J., Postek, M.T., Bennett, J., Modification of Hydrogen-Passivated Silicon by A Scanning Tunneling Microscope Operating in Air, Appl. Phys. Lett., 56(20), 20012003, 1990.

[2] Day, H.C., Allee, D.R., Selective Area Oxidation of Silicon With A Scanning Force Microscope, Appl. Phys. Lett., 61(21), 2691-2693, 1993.

[3] Namazu, T., Isono, Y., Tanaka, T., Evaluation of Size Effect on Mechanical Properties of Single Crystal Silicon by Nano-Scale Bending Test Using AFM, J. Microelectromech. Syst., 9(4), 450-459, 2000.

[4] Sugimura, H., Okiguchi, K., Nakagiri, N., Miyashita, M., Nanoscale Patterning of An Organosilane Monolayer on The Basis of Tip-Induced Electrochemistry in Atomic Force Microscopy, J. Vac. Sci. Technol. B, 14(6), 4140-4143, 1996.

[5] Ishibashi, M., Heike, S., Kajiyama, H., Wada, Y., Hashizume, T., Characteristics of Nanoscale Lithography Using AFM With A CurrentControlled Exposure System, Jpn. J. Appl. Phys., 37(3B), 1565-1569, 1998.

[6] Onoda, M., Tanaka, B., Matsuzuka, N., Isono, Y., Establishment of Probe Nano Writing Technique Using EB Resist and Application to Nano Devices, Proc. of the 23th Sensor Symposium on Sensors, Micromachines and Applied Systems, Takamatsu, Kagawa, Japan, 429432, October, 2006.

[7] Minne, S.C., Yaralioglu, G., Manalis, S.R., Adams, J.D., Zesch, J., Atalar, A., Quate, C.F., Automated Parallel High-Speed Atomic Force Microscopy, Appl. Phys. Lett., 73(12), 2340-2342, 1998.

[8] Matsuzuka, N., Tanaka, B., Nagamura, T., Sasaki, T., Namazu, T., Isono, Y., Development of Scanning Probe Parallel Nanowriting System With Electron Beam Resist, Proc. of the 14th Int. Conf. on Solid-State Sensors, Actuators and Microsystems (Transducers2007), Lyon, France, 16491652, June, 2007.

[9] Technical report ZEP520A, High Resolution Positive Electron Beam Resist, ZEON Corporation Specialty Materials Division, 2003. 